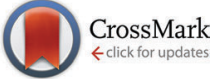

Cite this: Chem. Commun., 2015, 51, 2617

Received 24th November 2014, Accepted 23rd December 2014

DOI: $10.1039 / c 4 c c 09361 \mathrm{e}$

www.rsc.org/chemcomm

\section{Geminal dihalogen isosteric replacement in hydrated Al-2 affords potent quorum sensing modulators $\dagger$}

\author{
Min Guo, ${ }^{a}$ Yue Zheng, ${ }^{a}$ Jessica L. Terell, ${ }^{\mathrm{b}}$ Michal Ad, ${ }^{a}$ Clement Opoku-Temeng, ${ }^{a}$ \\ William E. Bentley and Herman O. Sintim*a
}

\begin{abstract}
Hydrated carbonyl groups in Al-2, a quorum sensing autoinducer, make key hydrogen bonding interactions in the binding site of LsrR (a transcriptional regulator). This can be recapitulated with geminal dibromides, via halogen bonding. Geminal dihalogens represent interesting isosteric replacements for hydrated carbonyls in ligands and are currently under-utilized in ligand design.
\end{abstract}

Quorum sensing in bacteria controls many processes, ranging from virulence factors production, pigmentation, biofilm formation, amongst others. ${ }^{1}$ The QS process starts with the production of signal molecules called autoinducers (AIs) by QS synthases, followed by exportation into the extracellular media and the perception of the signal by either membrane bound receptors (e.g. LuxP in vibrios) or intracellular (e.g. LuxR-type) QS receptors. The binding of autoinducers to these receptors ultimately leads to the modulation of transcriptional activators or repressors. Although many classes of QS autoinducers exist, the three main ones that have been actively studied are the Gram-positive peptides, ${ }^{2}$ Gram-negative acyl homoserine lactones ${ }^{3}$ and AI-2 (a mixture of interconverting isomers, see Fig. 1), which is used by both Gram-negative and Gram-positive bacteria. ${ }^{4}$

AI-2 is a complex and fascinating QS molecule. It is produced by many bacterial strains and either the molecule itself or its synthase, LuxS, affects the physiology (including biofilm formation $)^{1 b, 5}$ of multitudes of bacteria, some of which are of clinical and bioterrorism relevance, such as Vibrio cholera, ${ }^{6}$ Yersinia pestis ${ }^{7}$ and Staphyloccocus aureus. ${ }^{8}$ AI-2 production, as well as its degradation, affects central metabolism and emerging data suggest that it could be used as an alternative carbon source to produce acetyl-CoA. ${ }^{9}$ From a chemical perspective,

\footnotetext{
${ }^{a}$ Department of Chemistry and Biochemistry, University of Maryland College Park, 20742, USA. E-mail: hsintim@umd.edu; Fax: +1 301314 9121; Tel: +1 3014050633

${ }^{b}$ Fischell Department of Bioengineering, University of Maryland, College Park, 20742, USA

$\dagger$ Electronic supplementary information (ESI) available: Experimental details including synthesis, NMR spectra, mass and microbiological methods. See DOI: 10.1039/c4cc09361e
}

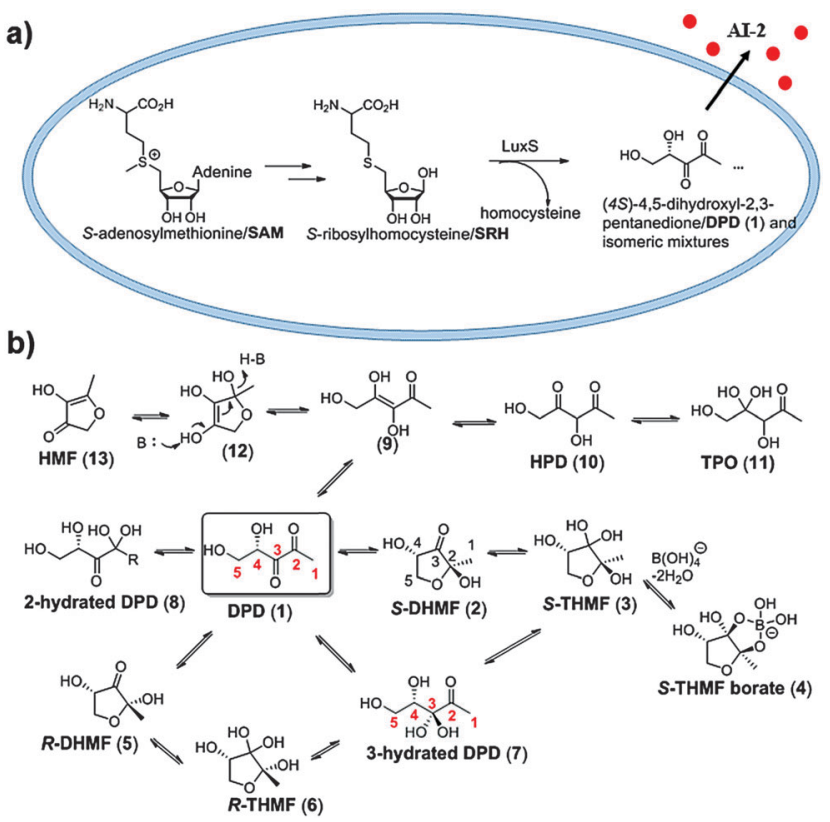

Fig. 1 (a) Biosynthesis of Al-2. (b) Chemical structures of Al-2.

AI-2 shows remarkable diversity as a signaling molecule (see Fig. 1 for the possible inter-converting isomers of AI-2) and depending on environmental conditions, AI-2 can exhibit selectivity in QS signaling. For example, in the presence of boric acid (such as in aquatic environments), AI-2 predominantly forms the borate ester (4, Fig. 1), which is a ligand for LuxP of vibrios. ${ }^{4}$ Enteric bacteria, such as E. coli and Salmonella, use AI-2, particularly after it has been processed by LsrK kinase. ${ }^{1 b, 10}$ So far four different proteins that bind to AI-2 have been characterized: LuxP, which binds to a borate form (4); ${ }^{4}$ LsrB (a transporter), which binds to the cyclic but nonborate form (6); ${ }^{11}$ LsrR, which binds to the phosphorylated linear form (and with the 3-position as the hydrate, 7) ${ }^{12}$ and LsrF, which binds to the 1,3-diketo form of AI-2 (10). ${ }^{9}$

Due to the ubiquity of AI-2 in bacterial QS signaling, there is interest in developing small molecules that can modulate 
AI-2-based signaling. ${ }^{13}$ Anti-AI-2 small molecules (or AI-2 receptor antagonists) could be used in synergy with traditional antibiotics. ${ }^{14}$ AI-2-based agonists have the potential to be used as tools in synthetic biology to modulate systems that use AI-2-based circuits. ${ }^{15}$ Fruitful approaches to develop anti-AI-2 molecules have been the modifications of $\mathrm{AI}-2$ at the $\mathrm{C} 1,^{13 a, 15,16} \mathrm{C} 4^{16 c, 17}$ or C5-positions ${ }^{13 f}$ (see Fig. 1, DPD 1 or 7, for the carbon labeling) into analogs that have shown great promise as either anti-biofilm or synergistic antibacterial agents. ${ }^{14}$ LsrR is a response regulator that represses the transcription of many genes, including biofilm-related genes. Upon binding to phosphorylated AI-2, LsrR no longer binds to DNA and hence biofilm and virulence-related genes can be transcribed. Recently we revealed that LsrR actually bound to the 3-hydrated form of AI-2 (7, Fig. 1). ${ }^{12}$ We rationalized that isosteric replacement at the C3 position of AI-2 could prevent isomerization into the 1,3-diketo analog (10), which is a substrate for LsrF degradation (see Fig. 2b). ${ }^{9}$

Analysis of interactions between P-AI-2 (phospho-3-hydrated DPD) and active-site residues in LsrR indicated that Asp243 was within $3 \AA$ of the geminal diol unit of AI-2 (see Fig. 3), indicating that if Asp243 existed as the carboxylate form, then the hydrogens of the geminal diol could potentially form hydrogen-bonding interactions with this residue. A geminal bromide or chloride, but not fluoride, could recapitulate this interaction via the halogen bond (the strength of the halogen bond is as follows: $\mathrm{I}>\mathrm{Br}>\mathrm{Cl} \gg \mathrm{F}) .{ }^{18}$ On the other hand, if the Asp243 is protonated in the active site, then it is conceivable that the carbonyl moiety could still partake in a halogen bond interaction with one of the geminal halogens whereas the carboxylic acid $\mathrm{OH}$ group would act as a hydrogen bond donor to the halogen. ${ }^{19}$ It is the ambivalence of higher halogens (presence of lone pairs for hydrogen bond formation and presence of a low lying $\sigma^{*}{ }_{\mathrm{C}-\mathrm{X}}$, see Fig. S1, ESI $\dagger$ or sigma hole for halogen bond formation $)^{20}$ that makes them ideal for isosteric replacement for the geminal diol.

Attempted synthesis of targeted dihalogen compounds 15-20 was fraught with difficulties because the final products were volatile (see ESI, $\uparrow$ Scheme S1). Others and us have, however, demonstrated that ester "prodrug" versions of AI-2 are convenient sources of AI- 2 because the esters can be easily purified using column

a)

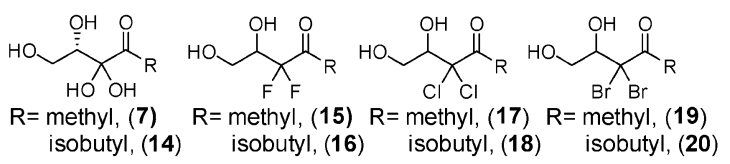

b)

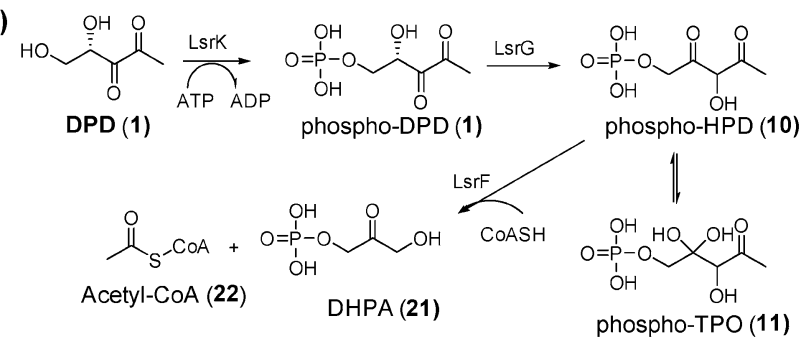

Fig. 2 (a) 3-hydrated DPD (7) and 3,3-dihalogen analogs. (b) Degradation of Al-2 via LsrG/LsrF. We expect that the geminal dihalogen analogs cannot form the 1,3-diketone intermediate (10) hence would not be degraded by LsrG/LsrF.
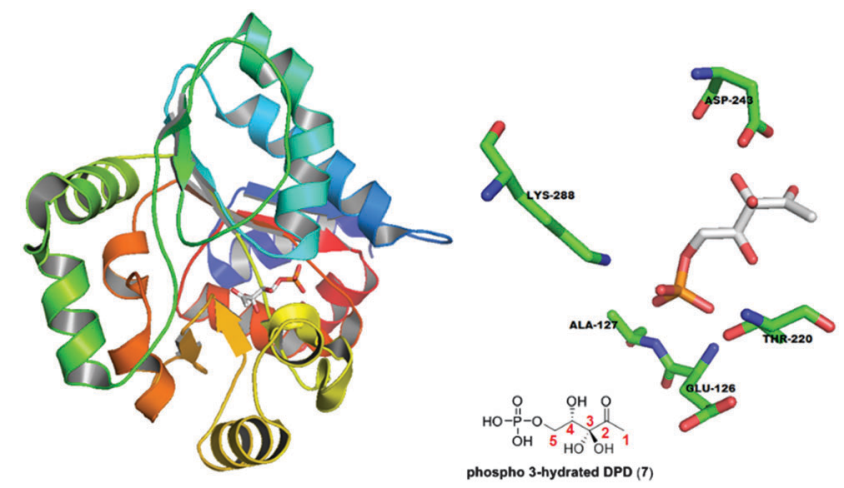

Fig. 3 Phospho-Al-2 in its hydrated form at C3 when binding with LsrR (PDB code: 4L4Z). Asp243 is $2.7 \AA$ from the geminal diol unit of phospho-Al-2.

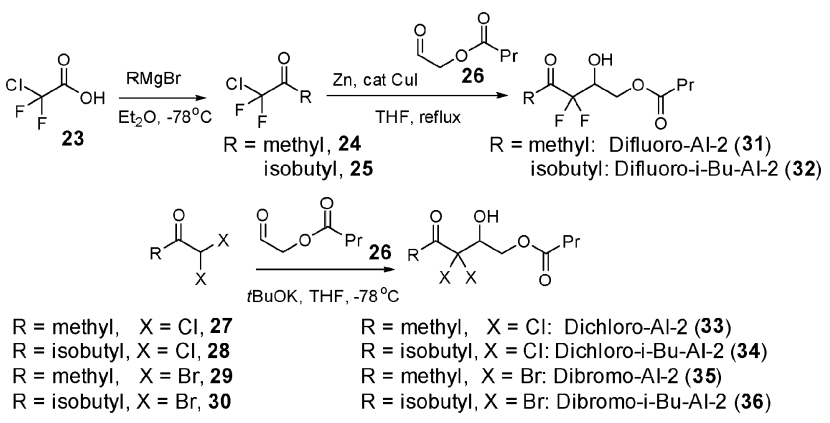

Scheme 1 Synthesis of dihalogenated Al-2 analogs.

chromatography and used directly in QS assays without prior deprotection of the esters. ${ }^{13 b, 21}$ Presumably, esterases produced by bacteria hydrolyze the esters into the active compounds, obviating a need for ester unmasking prior to administration. Therefore we proceeded to make the ester "pro-drug" versions of compounds 15-20 as 31-36. Target compounds, 31-36 were synthesized using strategies shown in Scheme 1.

With these molecules in hand, we investigated if they were nontoxic and could modulate quorum sensing in E. coli. Pleasingly, at $100 \mu \mathrm{M}$ concentration, all of the compounds were non-toxic towards E. coli (see Fig. S2, ESI $\dagger$ ). Using the E. coli strain LW7 (LuxS ${ }^{-}$and harboring the $\beta$-gal gene), we could use the $\beta$-galactosidase assay (see ESI, $\dagger$ Fig. S4) to evaluate our analogs. ${ }^{15,16}$ The $\beta$-gal gene in LW7 is under the control of the LsrR repressor. At high concentration of P-AI-2, the ligand binds to LsrR to dissociate it from the LsrR promoter region, resulting in the transcription of the genes under the control of the LsrR repressor. ${ }^{22}$ We have previously reported that the size of the $\mathrm{C} 1$ alkyl group of $\mathrm{AI}-2$ and analogs determines whether an analog would be an LsrR agonist or antagonist. ${ }^{16 a}$ Recently a rationale for this observation was provided via the crystal structure of LsrR in complex with AI-2 or analogs. ${ }^{12}$ Based on the LsrR crystal structure analysis, we postulated that the C1 methyl dihalogen analogs would act as agonists whereas the isobutyl dihalogen analogs would be antagonists, as long as the isosteric replacement of the geminal hydroxyl group with halogens did not adversely affect binding of the ligand to LsrR. Interestingly dibromoAI-2 (35) and dichloro-AI-2 (33), but not difluoro-AI-2 (31), could 
a)

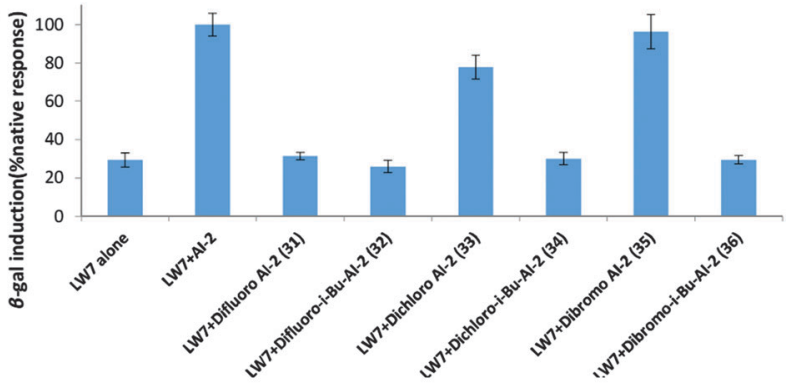

b)

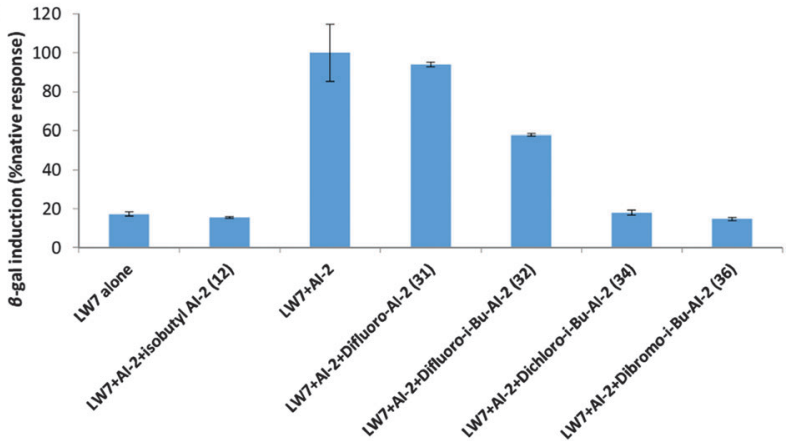

Fig. 4 (a) Al-2 dependent $\beta$-galactosidase production in E. coli LW7 (luxS ${ }^{-}$), in response to $20 \mu \mathrm{M}$ synthetic Al-2 or analogs. (b) Analogs (20 $\left.\mu \mathrm{M}\right)$ inhibit native $\beta$-galactosidase production in E. coli LW7 (luxS $^{-}$) in the presence of $20 \mu \mathrm{M}$ synthetic Al-2.

induce $\beta$-gal transcription (analyzed via the $\beta$-galactosidase assay), see Fig. 4a. In the absence of AI-2, the level of $\beta$-galactosidase is low whereas when $20 \mu \mathrm{M}$ of AI-2 or dibromo-AI-2 (35) or dichloro-AI-2 were added (33) to LW7, the level of $\beta$-galactosidase increased (see activity assay in Fig. 4a). Whereas the dibromo analog of AI-2 (35) was as potent as AI-2, the activity of the dichloro-AI-2 (33) was slightly lower than that of the native AI-2 molecule. In line with our expectation, none of the isobutyl dihalogen analogs were LsrR agonists, augmenting earlier observations that longer $\mathrm{C} 1$ chain $\mathrm{AI}-2$ analogs are not agonists but are rather antagonists. ${ }^{12,13,15,16}$

Isobutyl AI-2 (14) is a potent inhibitor of AI-2 signaling, via LsrR binding, ${ }^{12,15,16}$ and has been shown to inhibit E. coli biofilm formation, either alone or in combination with traditional antibiotics, such as gentamicin. ${ }^{14}$ In fact isobutyl AI-2 (14) has a higher affinity for LsrR than AI-2; the dissociation constant, $K_{\mathrm{d}}$, of the $\mathrm{AI}-2 / \mathrm{LsrR}$ complex is $2.0 \mu \mathrm{M}$ whereas isobutyl-AI-2/LsrR has a $K_{\mathrm{d}}$ of $0.5 \mu \mathrm{M}^{12}$ Since the dichloro and dibromo mimics of AI-2 $(33,35)$ were potent LsrR agonists, we wondered if the isobutyl-dihalogens would also be potent antagonists of LsrR. Interestingly both isobutyl dibromo and dichloro analogs of AI-2 were antagonists of LsrR (see Fig. 4b). Whereas in the absence of these ligands $20 \mu \mathrm{M}$ $\mathrm{AI}-2$ could induce $\beta$-gal expression, when equimolar amounts of isobutyl dibromo (36) or dichloro AI-2 (34) were added, these could compete with AI- 2 and prevent $\beta$-gal expression above the background, see Fig. 4b. Here too the isobutyl difluoro analog (32) was not an effective antagonist.

Next, we examined the effects of our analogs on E. coli W3110 pCT6 $\left(\mathrm{luxS}^{+}\right.$), which contains an egfp gene under LsrR control (also see ESI $\dagger$ ). Because W3110 pCT6 can make its own AI-2, in the absence of any AI-2 antagonist, about $90 \%$ of the population
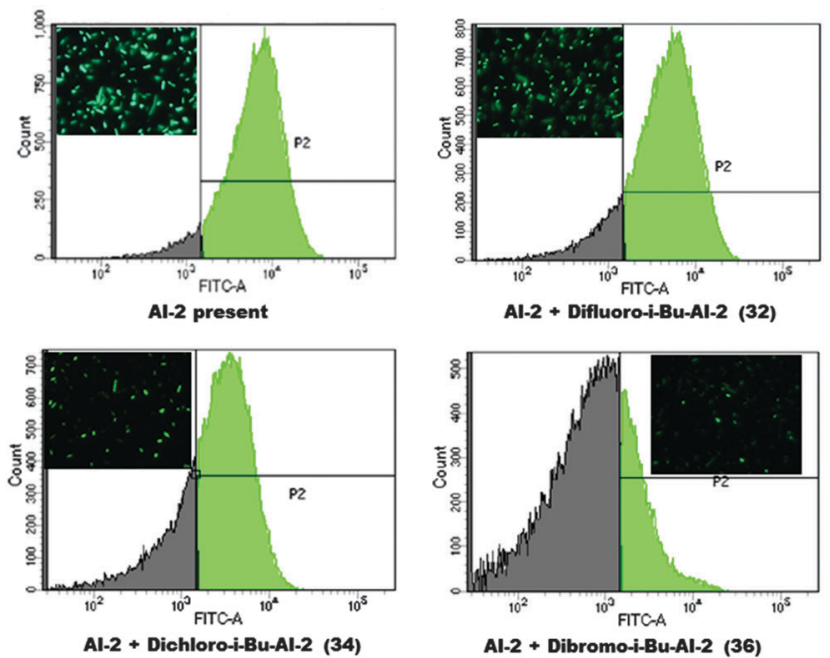

Fig. $5 \mathrm{Al}-2$ dependent EGFP induction in E. coli W3110 pCT6(luxS $\left.{ }^{+}\right)$in response to difluoro-i-Bu-Al-2 (32), dichloro-i-Bu-Al-2 (34) and dibromoi-Bu-Al-2 (36) (FACS analysis with microscopic image).

expressed the EGFP protein, see Fig. 5. The addition of the dihalogenated isobutyl DPD analogs caused a reduction of the EGFP expression. In agreement with the $\beta$-gal assay (Fig. 4), dibromoiBu-AI-2 (36) was a better inhibitor than dichloro-iBu-AI-2 (34), which in turn was better than difluoro-iBu-AI-2 (32) (see Fig. 5).

To explain our observations that the dibromo analog was a better mimic of hydrated AI-2 than the dichloro analog, which was also better than the difluoro analog, we compared the size (sterics) and electronics of the geminal dihydroxyl moiety with the various dihalogens in the cyclized and linear analog forms (see Fig. S5 and Table S1, ESI $\dagger$ ). The space-filling models of AI-2 and its halogenated analogs (Fig. S5, ESI $\dagger$ ) revealed that the fluoro analog (15) was smaller in size compared with AI-2 DPD (7) while both the chloro and bromo analogs, (17) and (19), were bigger than AI-2 DPD (7) (see Fig. S5 and also Table S1, ESI $\dagger$ ).

Apart from size, electronics could also play an important role in ligand-receptor binding. Electrostatic potential surfaces for AI-2 and analogs (Fig. 6 and Fig. S6, ESI $\dagger$ ), in both linear and cyclic forms were calculated using Gaussian $09^{23}$ at the B3LYP/6-31G(d) level with solvent effect (water, PCM model). For chloro- and bromo-analogs, in both linear and cyclized forms, there is a region of positive potential at the opposite end of the $\mathrm{C}-\mathrm{X}$ bond (on the halogen side). This positive region is called $\sigma$-hole, which is the basis of halogen bonding. So we speculate that chloro and bromo moieties in our analogs could function as electrophiles and partake in halogen bonding. Therefore even in the absence of hydroxyl groups, they could still interact with aspartic acid 243 in LsrR (see Fig. 3 and Fig. S1, ESI $\dagger$ ). In the case of difluoro analogs, neither the size of the fluoro moiety nor the electronics mimic the hydroxyl group well and hence the lack of activity (agonism or antagonism) seen with the difluoro substitution. An alternative explanation for the lack of activity of the difluoro analog could be the hydration of the $\mathrm{C} 2$ carbonyl group $(100 \%)$ in the difluoro analog, due to the superior electronwithdrawing nature of fluorine, compared to the other halogens. 

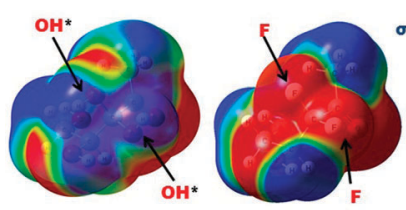

$\overbrace{\mathrm{OH}}^{9.5 \mathrm{kcal} / \mathrm{mol}} \prod_{7}^{\mathrm{HO}}$

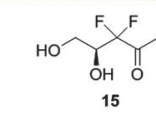

15

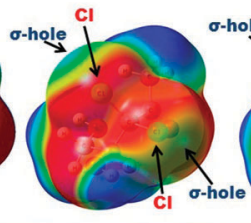

$9.5 \mathrm{kcal} / \mathrm{mol}$

$\mathrm{Br}-\mathrm{Br}$ HO 19

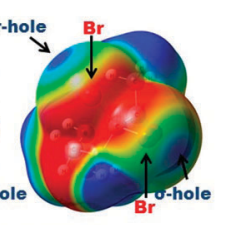

Fig. 6 Molecular surface electrostatic potential of Al-2 and analogs. Color ranges from $-9.5 \mathrm{kcal} \mathrm{mol}^{-1}$ (red) to $9.5 \mathrm{kcal} \mathrm{mol}^{-1}$ (blue). Important atoms and $\sigma$-hole are labeled. The $\sigma$-hole is a positively charged region and halogen bonding via this region is electrostatic in nature, distinct from the orbital picture given in Fig. S1, ESI. $\dagger$ Halogen bonding could have components of electrostatics and orbital-orbital interactions.

13-Carbon NMR, in the presence of water, of the fluoro analog reveals carbonyl peaks (see Fig. S3, ESI $\dagger$ ) and hence does not fully support this argument.

In conclusion, we have designed and prepared a new generation of AI-2 analogs with dihalogen at the C3 position. This set of analogs exhibited similar bioactivities with our earlier generation of analogs with modified $\mathrm{C} 1$ but intact $\mathrm{C} 3$ but has the added advantage that isomerization into a 1,3-diketo, which facilitates LsrF degradation, is not possible. This work demonstrates that geminal dihalogens, especially of higher halogens, are good mimics of hydrated moieties in biological ligands. It is expected that the substitution of geminal hydroxyl groups with dihalogen would afford molecules that could cross cell membranes more easily. Also for ligands whereby the hydrated form or keto form could facilitate degradation, such as in AI-2, isosteric replacement with dihalogens would provide more stable analogs. Geminal dihalogen-AI-2 adds to the increasing toolkit of small molecules that regulate bacterial phenotype. ${ }^{24}$

We are grateful to NSF (CBET 1264509) and Camille Dreyfus Founbdation (Teacher-Scholar fellowship to HOS) for funding. Yue Zheng is supported by Kraybill biochemistry fellowship and Ad Michal is supported by HHMI grant.

\section{Notes and references}

1 (a) M. B. Miller and B. L. Bassler, Annu. Rev. Microbiol., 2001, 55, 165-199; (b) J. Li, C. Attila, L. Wang, T. K. Wood, J. J. Valdes and W. E. Bentley, J. Bacteriol., 2007, 189, 6011-6020.

2 A. Tomasz and J. L. Mosser, Proc. Natl. Acad. Sci. U. S. A., 1966, 55, 58-66.

3 A. Eberhard, A. L. Burlingame, C. Eberhard, G. L. Kenyon, K. H. Nealson and N. J. Oppenheimer, Biochemistry, 1981, 20, 2444-2449.

$4 \mathrm{X}$. Chen, S. Schauder, N. Potier, A. Van Dorsselaer, I. Pelczer, B. L. Bassler and F. M. Hughson, Nature, 2002, 415, 545-549.
5 P. E. Kolenbrander, R. J. Palmer, Jr., S. Periasamy and N. S. Jakubovics, Nat. Rev. Microbiol., 2010, 8, 471-480.

6 M. B. Miller, K. Skorupski, D. H. Lenz, R. K. Taylor and B. L. Bassler, Cell, 2002, 110, 303-314.

7 J. Yu, M. L. Madsen, M. D. Carruthers, G. J. Phillips, J. S. Kavanaugh, J. M. Boyd, A. R. Horswill and F. C. Minion, Infect. Immun., 2013, 81, 4053-4062.

8 M. Li, A. E. Villaruz, V. Vadyvaloo, D. E. Sturdevant and M. Otto, BMC Microbiol., 2008, 8, 4.

9 J. C. Marques, I. K. Oh, D. C. Ly, P. Lamosa, M. R. Ventura, S. T. Miller and K. B. Xavier, Proc. Natl. Acad. Sci. U. S. A., 2014, 111, 14235-14240.

10 K. B. Xavier, S. T. Miller, W. Lu, J. H. Kim, J. Rabinowitz, I. Pelczer, M. F. Semmelhack and B. L. Bassler, ACS Chem. Biol., 2007, 2, 128-136.

11 (a) S. T. Miller, K. B. Xavier, S. R. Campagna, M. E. Taga, M. F. Semmelhack, B. L. Bassler and F. M. Hughson, Mol. Cell, 2004, 15, 677-687; (b) J. S. Kavanaugh, L. Gakhar and A. R. Horswill, Acta Crystallogr., Sect. F: Struct. Biol. Cryst. Commun., 2011, 67, 1501-1505.

12 J. H. Ha, Y. Eo, A. Grishaev, M. Guo, J. A. Smith, H. O. Sintim, E. H. Kim, H. K. Cheong, W. E. Bentley and K. S. Ryu, J. Am. Chem. Soc., 2013, 135, 15526-15535.

13 (a) C. A. Lowery, J. Park, G. F. Kaufmann and K. D. Janda, J. Am. Chem. Soc., 2008, 130, 9200-9201; (b) M. Guo, S. Gamby, S. Nakayama, J. Smith and H. O. Sintim, Sensors, 2012, 12, 3762-3772; (c) J. A. Gutierrez, T. Crowder, A. Rinaldo-Matthis, M. C. Ho, S. C. Almo and V. L. Schramm, Nat. Chem. Biol., 2009, 5, 251-257; (d) J. F. Alfaro, T. Zhang, D. P. Wynn, E. L. Karschner and Z. S. Zhou, Org. Lett., 2004, 6, 3043-3046; (e) T. Zang, B. W. Lee, L. M. Cannon, K. A. Ritter, S. Dai, D. Ren, T. K. Wood and Z. S. Zhou, Bioorg. Med. Chem. Lett., 2009, 19, 6200-6204; $(f)$ F. Rui, J. C. Marques, S. T. Miller, C. D. Maycock, K. B. Xavier and M. R. Ventura, Bioorg. Med. Chem., 2012, 20, 249-256; $(g)$ G. Brackman, A. A. Al Quntar, C. D. Enk, I. Karalic, H. J. Nelis, S. Van Calenbergh, M. Srebnik and T. Coenye, Bioorg. Med. Chem., 2013, 21, 660-667; (h) M. Li, N. Ni, H. T. Chou, C. D. Lu, P. C. Tai and B. Wang, ChemMedChem, 2008, 3, 1242-1249; (i) V. L. Malladi, A. J. Sobczak, T. M. Meyer, D. Pei and S. F. Wnuk, Bioorg. Med. Chem., 2011, 19, 5507-5519; $(j)$ V. Roy, B. L. Adams and W. E. Bentley, Enzyme Microb. Technol., 2011, 49, 113-123; (k) D. N. Quan and W. E. Bentley, PLoS Comput. Biol., 2012, 8, e1002637; (l) H. O. Sintim, J. A. I. Smith, J. Wang, S. Nakayama and L. Yan, Future Med. Chem., 2010, 2, 1005-1035.

14 V. Roy, M. T. Meyer, J. A. Smith, S. Gamby, H. O. Sintim, R. Ghodssi and W. E. Bentley, Appl. Microbiol. Biotechnol., 2012, 97, 2627-2638.

15 S. Gamby, V. Roy, M. Guo, J. A. Smith, J. Wang, J. E. Stewart, X. Wang, W. E. Bentley and H. O. Sintim, ACS Chem. Biol., 2012, 7, 1023-1030.

16 (a) V. Roy, J. A. Smith, J. Wang, J. E. Stewart, W. E. Bentley and H. O. Sintim, J. Am. Chem. Soc., 2010, 132, 11141-11150; (b) J. A. Smith, J. Wang, S. M. Nguyen-Mau, V. Lee and H. O. Sintim, Chem. Commun., 2009, 7033-7035; (c) K. Tsuchikama, J. Zhu, C. A. Lowery, G. F. Kaufmann and K. D. Janda, J. Am. Chem. Soc., 2012, 134, 13562-13564.

17 M. Kadirvel, F. Fanimarvasti, S. Forbes, A. McBain, J. M. Gardiner, G. D. Brown and S. Freeman, Chem. Commun., 2014, 50, 5000-5002.

18 P. Metrangolo and G. Resnati, Chem. - Eur. J., 2001, 7, 2511-2519.

19 E. T. Kool and H. O. Sintim, Chem. Commun., 2006, 3665-3675.

20 K. E. Riley, J. Rezac and P. Hobza, J. Mol. Model., 2013, 19, 2879-2883.

21 M. Frezza, L. Soulere, D. Balestrino, M. Gohar, C. Deshayes, Y. Queneau, C. Forestier and A. Doutheau, Bioorg. Med. Chem. Lett., $2007,17,1428-1431$.

22 M. E. Taga, S. T. Miller and B. L. Bassler, Mol. Microbiol., 2003, 50, 1411-1427.

23 Gaussian 09, Gaussian, Inc., Wallingford, CT, USA, 2009.

24 (a) R. J. Melander, M. J. Minvielle and C. Melander, Tetrahedron, 2014, 70, 6363-6372; (b) R. J. Worthington, M. S. Blackledge and C. Melander, Future Med. Chem., 2013, 5, 1265-1284. 